\title{
Integrable boundary conditions for nonlinear latticesi
}

\author{
I.T.Habibullin and A.N.Vil'danov
}

\begin{abstract}
Integrable boundary conditions in $1+1$ and $2+1$ dimensions are discussed from the higher symmetries point of view. Boundary conditions consistent with the discrete Landau-Lifshitz model and infinite 2D Toda lattice are represented.
\end{abstract}

\section{Introduction}

The inverse scattering transform method is a powerful tool for solving the Cauchy problem for nonlinear integrable equations. However the method is not sufficiently effective in application to the initial boundary value problems on a half line or a finite interval when both the boundary condition and the initial data are chosen arbitrary. At the same time there is a special kind of boundary conditions, called integrable, which are completely consistent with the integrability property of the equation. Such that the inverse scattering method is effectively applied to the initial boundary value problem when these boundary conditions are imposed. Several definitions of integrable boundary conditions are known in the literature (see, for instances, [1, 2]). In essence they are more or less equivalent. We will concentrate ourselves on one of them based on the symmetry approach.

During the last decade the classes of integrable boundary conditions have been studied for a large number of physically interesting

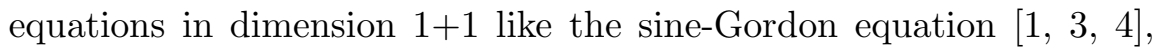
affine Toda lattices [5], the $\mathrm{KdV}$ equation [6] etc.

\footnotetext{
${ }^{1}$ Supported by RFBR, Grant \# 98-01-00576
} 
In the dimensions higher than $1+1$ the problem is still less studied. The main difficulty appearing in multi-dimensionality is connected with the so-called non-local dynamical variables higher symmetries and conserving quantities depend on. Recently [7] it has been shown that the symmetry approach can effectively be used to find integrable boundary conditions in $1+2$ dimensions.

In the present paper a boundary condition is found consistent with the Toda lattice by making use of the symmetry method. The boundary 2D lattice found reduced into the well-known boundary affine Toda field equations [5] by imposing the periodicity and similar other closure constraints.

Another model dealt with is the discrete version of the famous Landau-Lifshitz equation. Some integrable boundary conditions are represented for this chain. The boundary conditions found are differential constraints consistent with the nearest symmetry of the continuous L-L equation. An interesting fact is that under this constraint the symmetry mentioned turns into the Krichever-Novikov equation.

\section{Boundary condition for the discrete L-L model}

Let us consider an integrable lattice of the form

$$
u_{x}=f(u(n-1), u(n), u(n+1)), \quad-\infty<n<+\infty,
$$

where $u=u(n, x)$ is the unknown function and lower index denotes the derivative. Impose the following boundary condition at the point $n=k$

$$
u(k)=F(u(k+1), \ldots, u(k+m)) .
$$

Under this constraint the lattice (11) turns into a semi-infinite one, defined for all $n \geq k+1$. Let us given one more lattice of similar form

$$
u_{t}(n)=g(u(n-s), u(n-s+1), \ldots, u(n+s)),
$$


which is a symmetry of the lattice (1), i.e. two flows defined by chains (11) and (3) commute: $\left(u_{x}(n)\right)_{t}=\left(u_{t}(n)\right)_{x}$. It is clear that the constraint (11) and its differential consequences reduce the infinite chain (3) to a semi-infinite chain defined for $n \geq k+1$. The boundary condition (2) is called consistent with the symmetry (3), if these two semi-infinite lattices obtained are commuting.

If the boundary condition is consistent with an infinite set of symmetries of the lattice then it is called consistent with the integrability.

The commutativity of two semi-infinite lattices mentioned above might be checked directly but in some cases it is more convenient to utilize to this purpose the so called associated system. Let us rewrite the pair of equations (11), (3) as a system of partial differential equations. To this end introduce another set of dynamical variables, serving these chains and consisting of $u=u(k), v=u(k+1)$ and their $x$-derivatives $u_{x}, v_{x}, u_{x x}, v_{x x}, \ldots$ One expresses $u(n)$ for $n<k$ and $n>k+1$ through the new variables by using the equation (11) and its differential consequences. Substitute now the expressions obtained instead of the variables $u(n)$ into the chain (3). As a result one gets a system of the form

$$
\begin{aligned}
& u_{t}=g_{1}\left(u, v, u_{x}, v_{x}, \ldots, u_{s}\right), \\
& v_{t}=g_{2}\left(u, v, u_{x}, v_{x}, \ldots, v_{s}\right),
\end{aligned}
$$

where $u_{s}=\frac{\partial^{s} u}{\partial x^{s}}, v_{s}=\frac{\partial^{s} v}{\partial x^{s}}$. The boundary condition (2) reads as

$$
u=G\left(v, u_{x}, v_{x}, \ldots, u_{m}\right) .
$$

The following important statement is a consequence of these transforms [2].

Proposition. The boundary condition (2) is consistent with the symmetry (3) if and only if the constraint (5) is consistent with the associated system (雨).

It is clear that among the symmetries consistent with the integrable boundary condition one can find that of the smallest order. We refer 
to such a symmetry as a trial one. Our hypothesis, approved by numerous examples, claims that for the integrable equation given all trial symmetries connected with the certain type boundary conditions have one and the same order and this order can be pointed out a priori by rather simple preliminary analysis.

Let us consider an illustrative example. The following integrable discrete analogue of the well known Landau-Lifshits equation was found in [10] years ago:

$$
u_{n x}=v_{n}, \quad v_{n x}=-f_{n},
$$

where the function $f_{n}$ is defined as

$$
f_{n}=\left(v_{n}^{2}+P\left(u_{n}\right)\right)\left(\frac{1}{u_{n+1}-u_{n}}-\frac{1}{u_{n}-u_{n-1}}\right)+\frac{P^{\prime}\left(u_{n}\right)}{2},
$$

and $P(u)=a u^{4}+b u^{3}+c u^{2}+d u+e$ is an arbitrary polynomial of forth order with constant coefficients. The lattice (6) admits higher symmetries, the simplest one is of the form

$$
u_{n t}=h_{n}, \quad v_{n t}=D_{x}\left(h_{n}\right)
$$

where

$$
h_{n}=\left(v_{n}^{2}+P\left(u_{n}\right)\right)\left(\frac{1}{u_{n+1}-u_{n}}+\frac{1}{u_{n}-u_{n-1}}\right) .
$$

Let us introduce new variables $q=u_{-1}$ and $u=u_{0}$ and then by excluding the explicit $n$-dependence, as it was discussed above, bring the symmetry (7) to the form of associated system

$$
\begin{aligned}
u_{t} & =u_{x x}-2 \frac{u_{x}^{2}+P(u)}{u-q}+\frac{P^{\prime}(u)}{2}, \\
-q_{t} & =q_{x x}+2 \frac{q_{x}^{2}+P(q)}{u-q}+\frac{P^{\prime}(q)}{2},
\end{aligned}
$$

which is an integrable generalization of the Landau-Lifshits model. In the particular case, if the polynomial $P(u)$ subject to the additional constraint $P(u)=a u^{4}+c u^{2}+a$ then the system (8) coincides with the L-L model taken under the stereographic projection. The next 
symmetry of the chain (6) in terms of the variables $q, u$ is at the same time a symmetry of the system (8). We take it in the form of the associated system

$$
\begin{aligned}
& u_{\tau}=2 u_{x x x}+u_{x} P^{\prime \prime}(u)-\frac{12 u_{x}}{u-q}\left(u_{x x}+\frac{P^{\prime}(u)}{2}\right)+\frac{12 u_{x}}{(u-q)^{2}}\left(u_{x}^{2}+P(u)\right), \\
& q_{\tau}=2 q_{x x x}+q_{x} P^{\prime \prime}(q)+\frac{12 q_{x}}{u-q}\left(q_{x x}+\frac{P^{\prime}(q)}{2}\right)+\frac{12 q_{x}}{(u-q)^{2}}\left(q_{x}^{2}+P(q)\right) .(9)
\end{aligned}
$$

To find the boundary condition of the form

$$
u_{-1}=F\left(u_{0}\right)
$$

consistent with the integrability property of the Landau-Lifshits chain one has to answer the question when the constraint of the form $q=$ $H(u)$ is compatible with the trial symmetry (9). Only three choices are possible:

$$
\text { 1) } q=c, \quad 2) q=-u+c, \quad 3)\left(c_{1} q+c_{2}\right)\left(c_{1} u+c_{2}\right)=-1 \text {. }
$$

Here $c, c_{1}$, and $c_{2}$ are arbitrary constants.

\section{Higher dimensions}

In dimensions higher than $1+1$ the phenomenon of integrable boundary conditions is less studied. The classical generalized Toda lattices corresponding to infinite series of Lie algebras of finite growth can be interpreted as finite reductions of infinite 2D Toda lattice

$$
q_{x y}(n)=e^{q(n+1)-q(n)}-e^{q(n)-q(n-1)},
$$

with integrable cutting off conditions at two fixed points $n=0, n=N$. In [7] the question was examined when the boundary condition of the form

$$
q(1)=F\left(q(0), q_{x}(0), q_{y}(0), q(-1)\right)
$$


is consistent with the integrability property of the Toda lattice (11). In higher dimensions the main obstacle is connected with the nonlocal variables which higher symmetries depend on. For instance, the following two equations

$$
q_{t_{1}}(n)=b_{1}(n)+b_{1}(n-1)+q_{x}(n)^{2}
$$

and

$$
\begin{aligned}
q_{t}(n)= & b_{2}(n-2)+b_{2}(n-1)+b_{2}(n)+b_{1}(n)\left[2 q_{x}(n)+q_{x}(n+1)\right] \\
& +b_{1}(n-1)\left[2 q_{x}(n)+q_{x}(n-1)\right]+q_{x}(n)^{3}
\end{aligned}
$$

are two symmetries of the Toda lattice (11). They depend on two non-localities $b_{1}(n)$ and $b_{2}(n)$ which are introduced as solutions to the equations [8]:

$$
\begin{aligned}
q_{x x}(n) & =b_{1}(n)-b_{1}(n-1) \\
b_{1, y}(n) & =c(n)\left[q_{x}(n+1)-q_{x}(n)\right], \\
b_{1, x}(n) & =b_{1}(n)\left[q_{x}(n+1)-q_{x}(n)\right]+b_{2}(n)-b_{2}(n-1), \\
b_{2, y}(n) & =c(n) b_{1}(n+1)-c(n+1) b_{1}(n),
\end{aligned}
$$

where $c(n)$ is described by the equation $c(n)=e^{q(n+1)-q(n)}$. Other non-local variables $b_{j}, j>1$ satisfy the similar equations:

$$
\begin{aligned}
b_{j, x}(n) & =b_{j}(n)\left[q_{x}(n+j)-q_{x}(n)\right]+b_{j+1}(n)-b_{j+1}(n-1), \\
b_{j+1, y}(n) & =c(n) b_{j}(n+1)-c(n+j) b_{j}(n) .
\end{aligned}
$$

Let us pass from the standard set of local dynamical variables $q(n), q_{x}(n), q_{x x}(n), \ldots, q_{y}(n), q_{y y}(n), \ldots$ for all $n=0, \pm 1, \pm 2, \ldots$ to the set consisting of variables $u, v$ and their all $x$ - and $y$-derivatives by setting $u=e^{q(1)}, v=e^{-q(0)}$. For example, $q(-1)$ may be expressed from the Toda equation written in the form $e^{-q(-1)}=e^{q(1)-2 q(0)}-$ $q_{x y}(0) e^{-q(0)}$, and so on. In terms of these new variables the symmetries (13) and (14) become (see, also [8])

$$
u_{t_{1}}=u_{x x}+2 r u \quad, v_{t_{1}}=-v_{x x}-2 r v,
$$




$$
\begin{aligned}
& u_{t}=u_{x x x}+3 r u_{x}+3 s u \\
& v_{t}=v_{x x x}+3 r v_{x}-3 s v+3 r_{x} v
\end{aligned}
$$

where non-localities $r=b_{1}(0)$ and $s=b_{2}(0)+r(\log u)_{x}$ obviously satisfy the equations $r_{y}=(u v)_{x}, \quad s_{y}=\left(u_{x} v\right)_{x}$. The boundary condition (12) takes the form

$$
u=\tilde{F}\left(v, v_{x}, v_{y}, v_{x y}\right) .
$$

A very useful consequence of the change of variables is the following statement:

Proposition. The boundary condition (12) is compatible with the symmetry (14) (or (13)) if and only if the constraint (21) is consistent with the system (20) (or (19)).

Remark. The connection between the "new" and "old" dynamical variables has some singularities at points $u=0$ and $v=0$ because of the formulae $s=b_{2}(0)+r(\log u)_{x}$ and $s=b_{2}(-1)-r(\log v)_{x}+b_{1, x}(0)$. That is why these cases should be checked directly without passing to the associated system.

In [7] two constraints has been classified

$$
u=\tilde{F}\left(v, v_{x}, v_{y}, v_{x y}\right),
$$

and

$$
u_{y}=\tilde{G}\left(u, v, v_{x}\right) .
$$

In both cases the equation (20) was taken as the trial symmetry because the other one (19) does not admit any constraint of the above forms, except two degenerate ones $u=0$ and $v=0$ (see Remark above), for it is skew-symmetric in the highest order derivatives.

The consistency condition with the $t$-dynamics allows one to extract the following five choices for the function $\tilde{F}$, here we give also the additional constraint the non-localities have to satisfy to:

(i) $u=0, s_{y}=0$;

(ii) $u=a, s=0$;

(iii) $u=a v, s=\frac{1}{2} r_{x}$; 
(iv) $u=\frac{v_{x y}}{\left(a-v^{2}\right)}+\frac{v v_{x} v_{y}}{\left(a-v^{2}\right)^{2}}, \quad s=r_{x}-\frac{v_{x} v_{x x}}{a-v^{2}}-\frac{v v_{x}^{3}}{\left(a-v^{2}\right)^{2}}$;

here $a$ is an arbitrary constant,

(v) $u=-\frac{v_{x y}}{v^{2}}+\frac{v_{x} v_{y}}{v^{3}}, \quad r=-\frac{v_{x x}}{v}+\frac{v_{x}^{2}}{v^{2}}+b$

and $b$ is an arbitrary function of $x$.

Rewritten in terms of the basic variables these choices read:

(1) $e^{q(1)}=0, b_{2, y}(0)=0$;

(2) $q(1)=$ const.,$b_{2}(0)=0$;

(3) $q(1)=-q(0)+$ const., $b_{2}(0)=\frac{b_{1, x}(0)}{2}+b_{1}(0) q_{x}(0)$;

(4) $a e^{q(1)}=e^{-q(-1)}+\frac{a q_{x}(0) q_{y}(0)}{a e^{q(0)}-e^{-q(0)}}$,

$b_{2}(0)=b_{1, x}(0)-b_{1}(0) q_{x}(1)+\frac{a q_{x}(0)^{3}}{\left(a e^{q(0)}-e^{-q(0)}\right)^{2}}-\frac{q_{x}(0) q_{x x}(0) e^{-q(0)}}{a e^{q(0)}-e^{-q(0)}} ;$

(5) $e^{-q(-1)}=0, b_{1, y}(-1)=0$.

All these boundary conditions are known as integrable [9]. Among constraints of the form (23) the only is consistent with the trial symme$\operatorname{try}(20): \quad \tilde{G}=a v_{x}$, where $a \neq 0$ is an arbitrary constant (if $a=0$ then the constraint $u_{y}=0$ is reduced by integration to the form $u=$ const). Here non-localities should satisfy the constraint $s=u_{x}-\frac{u u_{x}}{a}$.

Turning back to the original variables yields the following boundary condition

$$
q_{y}(1)=-a e^{-q(1)-q(0)} q_{x}(0),
$$

which is not reduced to any of standard ones. So the following system of hyperbolic equations

$$
\begin{aligned}
q_{x}(0) & =-a_{1} e^{q(1)+q(0)} q_{y}(1), \\
q_{x y}(j) & =e^{q(j+1)-q(j)}-e^{q(j)-q(j-1)}, 1 \geq j \geq N, \\
q_{y}(N+1) & =-a_{N} e^{-q(N+1)-q(N)} q_{x}(N),
\end{aligned}
$$

is an integrable reduction of the 2D Toda lattice. Similarly one can reduce the Toda lattice (11) by imposing different kind closure constraints at the ends:

$$
q_{x}(0)=-a_{1} e^{q(1)+q(0)} q_{y}(1)
$$




$$
\begin{aligned}
q_{x y}(j) & =e^{q(j+1)-q(j)}-e^{q(j)-q(j-1)}, 1 \geq j \geq N, \\
q(N+1) & =\tilde{F}\left(q(N), q_{x}(N), q_{y}(N), q(N-1)\right),
\end{aligned}
$$

where $\tilde{F}$ is one of the boundary conditions (1)-(5) above. Undoubtedly the systems (25), (26) are integrable, but it is not clear what is the algebraic structure they are related to.

\section{Higher dimensions - boundary con- ditions of the other kind}

Now let us represent the Toda lattice as an infinite system of hyperbolic equations

$$
q_{t t}(n)-q_{z z}(n)=e^{q(n+1)-q(n)}-e^{q(n)-q(n-1)}, \quad-\infty<n<+\infty,
$$

where the new independent variables are introduced as follows $t=x+y$ $z=y-x$. Consider this system on the half-line $z>0$ imposing along the border $z=0$ a boundary condition of the form

$$
q_{z}(n)=H_{n}(q(n+1), q(n), q(n-1)), \quad-\infty<n<+\infty,
$$

The problem is now to extract from the class of boundary conditions (28) those consistent with the integrability. To solve the problem we will use the symmetry approach. First choose up the trial symmetry and rewrite it as an associated system. Notice that under the reflection type transformation $x \rightarrow y, y \rightarrow x$ the symmetries of the Toda lattice turn info symmetries, for the lattice itself is invariant under this transformation. So the symmetry (13), (15) produces a new one:

$q_{t_{2}}(n)=p_{1}(n)+p_{1}(n-1)+q_{y}(n)^{2}, p_{1}(n)=p_{1}(n-1)+q_{y y}(n)$. The sum of this symmetry and its counterpart (13), (15) is again a symmetry but now it is invariant under the $x, y^{\text {'reflection }} x \rightarrow y, y \rightarrow x$ :

$$
\begin{aligned}
q_{\tau}(n) & =h(n)+h(n-1)+q_{x}(n)^{2}+q_{y}(n)^{2}, \\
h(n) & =h(n-1)+q_{x x}(n)+q_{y y}(n),
\end{aligned}
$$


here $h(n)=p_{1}(n)+b_{1}(n)$. Notice that the symmetry $(29)$ can be reduced to the famous Davey-Stewardson equation. In terms of the new independent variables $t, z$ it reads

$$
\begin{aligned}
q_{\tau}(n) & =4 q_{t t}(n)-2 c(n)+2 c(n-1)+2 h(n-1)+2 q_{t}(n)^{2}+2 q_{z}(n)^{2}, \\
h(n) & =h(n-1)+4 q_{t t}(n)-2 c(n)+2 c(n-1) .
\end{aligned}
$$

This symmetry will be taken as a trial one. The dynamical set of variables serving the system (30) consists of the local variables $q(n), q_{z}(n)$ and their $t$-derivatives for all $n$ and non-local variables $h(n)$ and their $z$-derivatives. Let us put $v(n)=q_{z}(n), g(n)=h_{z}(n)$ and represent the symmetry (30) as an associated system

$$
\begin{aligned}
q_{\tau}(n)= & 4 q_{t t}(n)-2 c(n)+2 c(n-1)+2 h(n-1)+2 q_{t}(n)^{2}+2 v(n)^{2}, \\
v_{\tau}(n)= & 4 v_{t t}-2 c(n)(v(n+1)-v(n))+2 c(n-1)(v(n)-v(n-1))+ \\
& +2 g(n-1)+4 q_{t}(n) v_{t}(n)+4 v(n)\left(q_{t t}(n)-c(n)+c(n-1)\right), \\
h(n)= & h(n-1)+4 q_{t t}(n)-2 c(n)+2 c(n-1), \\
g(n)= & g(n-1)+4 v_{t t}-2 c(n)(v(n+1)-v(n))+ \\
& +2 c(n-1)(v(n)-v(n-1)) .
\end{aligned}
$$

The boundary condition (28) for the Toda lattice (27) is consistent with the trial symmetry (30) if and only if the differential constraint

$$
v(n)=H_{n}(q(n-1), q(n), q(n+1)) .
$$

is consistent with the system of equations (31). Direct computations show that only choice of $H$ is (remind that $v=q_{z}$ )

$$
\left.q_{z}(n)\right|_{z=0}=c_{n} e^{\frac{q(n+1)-q(n)}{2}}-c_{n-1} e^{\frac{q(n)-q(n-1)}{2}},
$$

where $c_{n}^{2}=1$ for all $n$, either $c_{n}=0$ for all $n$. Combining the periodicity closure constraint $q(n)=q(n+N)$ with the boundary condition (33) one gets a finite system of hyperbolic equations on a half-plane

$$
\begin{aligned}
& q_{t t}(n)-q_{z z}(n)=e^{q(n+1)-q(n)}-e^{q(n)-q(n-1)}, \quad 0<z<+\infty \\
& \left.q_{z}(n)\right|_{z=0}=c_{n} e^{\frac{q(n+1)-q(n)}{2}}-c_{n-1} e^{\frac{q(n)-q(n-1)}{2}} \\
& q(0)=q(N), \quad q(N+1)=q(1) .
\end{aligned}
$$


found recently by E.Corrigan et al. (see [5]). Imposing the closure conditions of the form (1)-(5) in addition to the boundary condition (33) leads again to integrable boundary value problem from [5]. This kind problems for finite Toda systems have beautiful interpretation in the field theory.

\section{References}

[1] E.K.Sklyanin, Funct. Analy. Appl. 21(1987), 164.

[2] B.Gürel, M.Gürses, I.Habibullin. J. Math. Phys. 1995. V. 36. \#12. P. 6809.

[3] R.Bikbaev, V.Tarasov, Algebra and Analysis. 1991, V.3, \#4, P.78.

[4] S.Ghoshal, A.B.Zamolodchikov, Int. J. Mat. Phys. A 9 (1994), 83.

[5] E.Corrigan, P.E.Dorey, R.H.Rietdijk, R.Sasaki, Phys. Letts B 333 (1994), 83.

[6] V.Adler, B.Gürel, M.Gürses, I.Habibullin. J. Phys. A: Math. Gen. 1997. V.30, P. 3505-3513.

[7] B.Gürel, I.Habibullin, Phys. Letts A, 233 (1997), 68-72.

[8] A.B.Shabat, Phys. Letts A, 200 (1995) 121.

[9] A.V.Mikhailov, M.A. Olshanetsky and A.M.Perelomov, Commun. Math. Phys. 79 (1981), 473.

[10] A.B.Shabat and R.I.Yamilov, Algebra and Analysis. 2:2 (1990), 183 